A $\mathrm{C}$ Gublications $\quad$ Rec. Nat. Prod. 13:1 (2018) 1-9

records of natural products

\title{
Phytochemical Changes in Aerial Parts of Hypericum perforatum at Different Harvest Stages
}

\author{
Ping Sun ${ }^{1} \oplus$, Tianlan Kang ${ }^{2} \oplus$, Hua Xing ${ }^{1} \oplus$, Zhen Zhang ${ }^{1}$, Delong Yang $^{1} \oplus$, \\ Jinlin Zhang ${ }^{1,3} \oplus$, Paul W. Paré ${ }^{4}$ and Mengfei $\mathrm{Li}^{1,4_{\odot}}{ }^{*}$
}

${ }^{1}$ Gansu Provincial Key Lab of Aridland Crop Science / College of Life Science and Technology, Gansu Agricultural University, 730070 Lanzhou, China

${ }^{2}$ Institute of Industrial Crops and Production, 730000 Lanzhou, China

${ }^{3}$ College of Pastoral Agriculture Science and Technology, Lanzhou University, 730000 Lanzhou, China

${ }^{4}$ Department of Chemistry and Biochemistry, Texas Tech University, 79409 Texas, USA

(Received April 04, 2018; Revised May 24, 2018; Accepted May 25, 2018)

\begin{abstract}
Hypericum perforatum is a perennial herb that produces anti-depression metabolite hypericin (Hyp) as well as flavonoids and polyphenols. In order to find the optimization of harvest stage to increase bioactive compounds production, the levels of Hyp, flavonoids and polyphenols as well as antioxidant capacity were evaluated by the assays of high performance liquid chromatography (HPLC), $\mathrm{NaNO}_{2}-\mathrm{AlCl}_{3}-\mathrm{NaOH}$ and FolinCiocalteu as well as 1, 1-diphenyl-1-picrylhydrazyl (DPPH) and ferric reducing antioxidant power (FRAP), respectively. The results showed that there was no difference in antioxidant capacity of extracts from aerial parts between floral budding stage (FBS) and blooming stage (BS), while significant decrease was observed at fruit set stage (FSS) compared to BS $(P<0.05)$. On a per plant basis, biomass and the levels of Hyp, flavonoids and polyphenols in aerial parts decreased during harvest stages, even if bioactive compounds contents in flower reached the highest at BS on a dry weight basis. These findings indicated that the optimization of harvest stage of $H$. perforatum should be at FBS.
\end{abstract}

Keywords: Hypericum perforatum; harvest stage; hypericin; flavonoids; polyphenols; antioxidant capacity. (C) 2018 ACG Publications. All rights reserved.

\section{Introduction}

Hypericum perforatum (family Hypericaceae), commonly known as St John's Wort, is a perennial herbaceous herb distributed in Europe, Northern Africa, Northern America and China [1,2]. Aerial parts are the major source of the highly valued polycyclic dianthroquinone-hypericin (Hyp), which is used worldwide for the treatment of mild to moderate depression [3]. Flavonoids (e.g. hyperoside, isoquercitrine and quercitrine) and polyphenols (e.g. chlorogenic acids, caffeic and coumaroylquinic

\footnotetext{
* Corresponding author: E-Mail: 1mf@gsau.edu.cn; Phone:086-931-7631742 Fax:086-931-7631742
}

The The article was published by ACG Publications 
acids) have also been identified in the aerial parts $[4,5]$; these compounds have shown several biological activities such as anti-oxidant, anti-viral and anti-microbial agents $[6,7]$.

Levels of Hyp and polyphenols in flower or leaves have been observed to be significantly affected by environmental factors such as drought, soil nutrients and harvest stage [7-10]. On a dry weight basis, Hyp reached the highest level at BS, while levels of monoflavonoids and biflavonoids maximized at FBS and BS, respectively $[7,11,12]$. Meanwhile, large variations in dry weight of aerial components were also observed during harvest stages [11].

Unlike previous studies that plants were chemically analyzed as fixed flower/leaf tissue on a dry weight basis [8, 10-12], in this study, effect of harvest stages on levels of Hyp, flavonoids, polyphenols and antioxidant capacity in aerial parts (stem, leaf and flower) was systematically investigated on both dry weight and per plant basis.

\section{Materials and Methods}

\subsection{Plant Materials}

Hypericum perforatum were cultivated in situ conditions in Kangxian ( $\left.33^{\circ} 16^{\prime} 20^{\prime \prime} \mathrm{N}, 105^{\circ} 31^{\prime} 50^{\prime \prime} \mathrm{E}\right)$ of Gansu Province, China in March, 2014. The aerial parts (upper 1/2) of 3-year-old plants were harvested in May, 2016 at three developmental stages including: floral budding stage (FBS), blooming stage (BS) and fruit set stage (FSS) (Figure S1). For each stage, plant samples (30 plants) were randomly collected and dried in a dark and ventilated room. No additional irrigation and fertilizer was applied in the experimental field. A voucher specimen (No. GAU-HP-001) was deposited in the herbarium of College of Life Science and Technology, Gansu Agricultural University, Lanzhou, China.

\subsection{Reagents}

DPPH, TPTZ (2, 4, 6-tris (2-pyridyl)-s-triazine), gallic acid and catechin were purchased from Sigma (USA). $\mathrm{AlCl}_{3}, \mathrm{FeCl}_{3} \cdot 6 \mathrm{H}_{2} \mathrm{O}, \mathrm{FeSO}_{4} \cdot 7 \mathrm{H}_{2} \mathrm{O}, \mathrm{Na}_{2} \mathrm{CO}_{3}, \mathrm{NaNO}_{2}, \mathrm{HCl}$, Folin-Ciocalteu, ethanol and methanol were purchased from Tianjin Guangfu Chemical Research Institute (Tianjin, China). All chemicals were of analytical grade.

\subsection{Preparation of Extracts}

Finely powdered samples $(2.0 \mathrm{~g})$ suspended in ethanol $(95 \%$ and $10 \% \mathrm{v} / \mathrm{v}, 50 \mathrm{~mL})$ were agitated in the dark for $24 \mathrm{~h}$ at $35^{\circ} \mathrm{C}$, then centrifuged at $8,000 \mathrm{rpm}$ for $10 \mathrm{~min}$ at $4^{\circ} \mathrm{C}$. Following exhaustive extraction $(\times 3)$, the extracts were combined and dried in vacuo at $35^{\circ} \mathrm{C}$. Dried residue was re-dissolved in ethanol $(95 \% \mathrm{v} / \mathrm{v})$ to $10 \mathrm{mg} / \mathrm{mL}$ concentration and filtered $(0.22 \mu \mathrm{m}$ durapore membrane; Millipore, Sigma, USA). Samples from different tissues were extracted in triplicate.

\subsection{Antioxidant Capacity}

Although several methods for evaluating antioxidant capacity of natural plant extracts, no perfect system is available to define the "true" level of a complex medium $[13,14]$. The DPPH and FRAP assays are widely used by many researchers for rapid evaluation of antioxidant $[15,16]$. For DPPH assay, it is one of the few stable and commercially available organic nitrogen radical assays via an electron transfer reaction, which can be measured by the decoloration assay where DPPH has an absorption band at $515 \mathrm{~nm}$ that disappears upon reduction by an antiradical compound $[17,18]$. For FRAP assay, reductants (antioxidants) reduce ferric-tripyridyltriazine complex $\left(\mathrm{Fe}^{3+}-\mathrm{TPTZ}\right)$ to a blue ferrous form $\left(\mathrm{Fe}^{2+}\right)$ with an increase in absorbance at $593 \mathrm{~nm}[19,20]$.

$D P P H$ radical scavenging assay: The free radical scavenging activity of DPPH was measured according to previous protocols $[16,21]$. Briefly, ethanol extract $(100 \mu \mathrm{L})$ was added into $10^{-4} \mathrm{~mol} / \mathrm{L}$ DPPH methanol solution $(2.9 \mathrm{~mL})$ then the mixture was shaken and kept in dark for $30 \mathrm{~min}$ at $25^{\circ} \mathrm{C}$. The absorbance was detected at $515 \mathrm{~nm}$. The capability to scavenge DPPH radicals was calculated as follows: 
DPPH scavenging activity $(\%)=\left[\left(A_{0}-A\right) / A_{0}\right] \times 100$

Where " $A$,"and " $A$ " were the absorbance of DPPH without and with sample, respectively.

FRAP assay: The capability to reduce ferric-tripyridyltriazine complex $\left(\mathrm{Fe}^{3+}-\mathrm{TPTZ}\right)$ was measured according to previous protocols $[16,21]$. Briefly, ethanol extracts $(100 \mu \mathrm{L})$ or standard samples $\left(\mathrm{FeSO}_{4} \cdot 7 \mathrm{H}_{2} \mathrm{O}, 500 \mu \mathrm{mol}\right)$ was added into FRAP reagent $(2.9 \mathrm{~mL})$ then the mixture was shaken and kept in dark for $5 \mathrm{~min}$ at $37^{\circ} \mathrm{C}$. The absorbance was detected at $593 \mathrm{~nm}$. The FRAP value was calculated on the basis of $500 \mu \mathrm{M} \mathrm{Fe}^{2+}\left(\mathrm{FeSO}_{4} \cdot 7 \mathrm{H}_{2} \mathrm{O}\right)$ as follows:

FRAP value $(\mu \mathrm{mol} \mathrm{Fe}(\mathrm{II}) / \mathrm{g})=\left(\Delta A_{593}\right.$ test sample $/ \Delta A_{593}$ standard sample $) \times 500(\mu \mathrm{mol} \mathrm{Fe}(\mathrm{II}) / \mathrm{g})$

Where $\Delta A_{593}$ was the absorbance of sample minus the absorbance of blank at $4^{\text {th }}$ minute.

\subsection{Hypericin HPLC Quantification}

Extract samples $(20 \mu \mathrm{L})$ were analyzed using a Nova Pack $\mathrm{C}_{18}$ column $(250 \times 4.6 \mathrm{~mm}, 5 \mu \mathrm{m})$ in a HPLC system (LC-10A, Shimadzu, Japan) based on a previous protocol [22] with modification on detection wavelength. The mobile phase consisted of $0.03 \mathrm{~mol} / \mathrm{L} \mathrm{KH}_{2} \mathrm{PO}_{4}$ adjusted to $\mathrm{pH} 7.0$ with 0.5 $\mathrm{mol} / \mathrm{L} \mathrm{K}_{2} \mathrm{HPO}_{4}$ and methanol $(30: 70, \mathrm{v} / \mathrm{v})$, the mobile phase was filtered and degassed prior to use. 2.0 $\mathrm{mg} / \mathrm{mL}$ The flow rate was $1.0 \mathrm{~mL} / \mathrm{min}$ and samples were detected at $254 \mathrm{~nm}$ with the injection volume $20 \mu \mathrm{L}$. The quantification based on peak area comparison with a reference standard curve using Hyp (56690; Sigma, USA) (Figure S2).

\subsection{Flavonoids Quantification}

Flavonoids content was determined based on $\mathrm{NaNO}_{2}-\mathrm{AlCl}_{3}-\mathrm{NaOH}$ method [16, 23]. Extract sample $(200 \mu \mathrm{L})$ was added into $\mathrm{ddH}_{2} \mathrm{O}(2 \mathrm{~mL})$ and $\mathrm{NaNO}_{2}(5 \%, 0.3 \mathrm{~mL})$; after the mixture agitating for $5 \mathrm{~min}$, $\mathrm{AlCl}_{3}(10 \%, 0.3 \mathrm{~mL})$ was added and reacted for $1 \mathrm{~min}$ at room temperature; then $\mathrm{NaOH}\left(1.0 \mathrm{~mol} \cdot \mathrm{L}^{-1}, 2\right.$ $\mathrm{mL}$ ) was added to stop the reaction; absorbance was detected at $510 \mathrm{~nm}$. All determinations were carried out in triplicate. Flavonoids content was expressed as $\mathrm{mg}$ of catechin equivalent (CE).

\subsection{Polyphenols Quantification}

Polyphenols content was determined based on Folin-Ciocalteu method [16, 23]. Extract sample (20 $\mu \mathrm{L})$ was added into Folin-Ciocalteu reagent $(10 \%, 2 \mathrm{~mL})$; after the mixture agitating for $5 \mathrm{~min}, \mathrm{Na}_{2} \mathrm{CO}_{3}$ $(7.5 \%, 1.6 \mathrm{~mL})$ was added and then reacted for $15 \mathrm{~min}$ at $37^{\circ} \mathrm{C}$; absorbance was detected at $725 \mathrm{~nm}$. All determinations were carried out in triplicate. Polyphenols content was expressed as mg of gallic acid equivalent (GAE).

\subsection{Statistical Analysis}

Statistical analysis was performed via a one-way analysis of variance and Duncan multiple comparison tests. SPSS 22.0 was the software package used with $P<0.05$ as the basis for statistical differences.

\section{Results and Discussion}

\subsection{Antioxidant Capacity at Different Harvest Stages}

Since one single method cannot be fully evaluated antioxidant capacity of natural antioxidants [24,25], DPPH and FRAP assays were selected due in large part to a significant positive correlation between DPPH scavenging activity and FRAP value $[15,16,26]$. As shown in Figure 1, a significant difference in antioxidant capacity of ethanol (95\% and 15\%) extracts from aerial parts (stem, leaf and flower) was observed at different harvest stages; in both DPPH and FRAP assays, 95\% ethanol extracts showed higher antioxidant capacity compared to that of 10\% ethanol; between FBS and BS, no 
significant difference was observed in aerial parts (stem, leaf and flower); however, a significant decrease in leaf and flower was observed at FSS compared to BS.

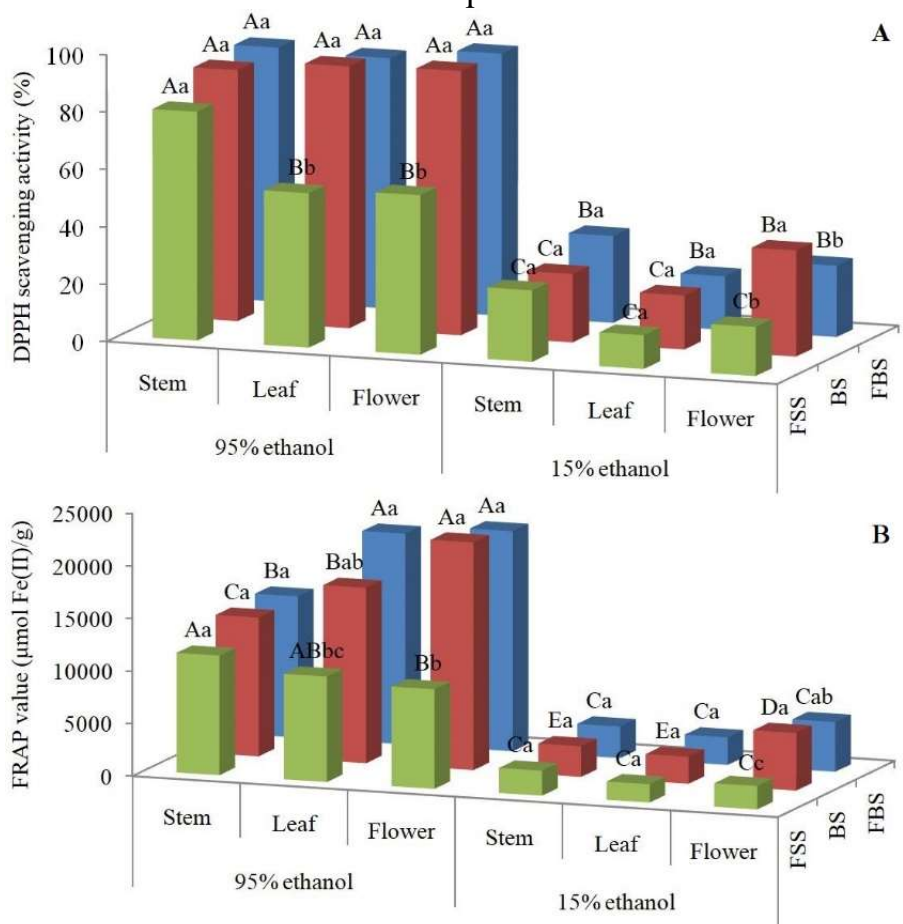

Figure 1. Antioxidant capacity of extracts from aerial parts of $H$. perforatum at different harvest stages, evaluated by DPPH (A) and FRAP (B) assays. Different lowercase letters indicate significant difference at $P<0.05$ for different harvest stages within the same tissue component. Different uppercase letters indicate significant difference at $P<0.05$ for different tissue components and solvents within the same harvest stage. The same as below.

\subsection{Aerial parts biomass at Different Harvest Stages}

As shown in Figure 2, there was no difference in the dry weight of stem and leaf between FBS and BS, while significant decrease was observed at FSS compared to BS; the flower dry weight peaked at BS and significantly greater than FBS and FSS. In a whole, the aerial parts biomass were 14.96, 14.97 and $12.54 \mathrm{~g} /$ plant at FBS, BS and FSS, respectively, which exhibited that there was no difference between FBS and BS and remarkable decrease at FSS. In addition, the proportion of stem: leaf: flower was observed to largely vary from $57: 36: 7 \%$ to $63: 36: 1 \%$.

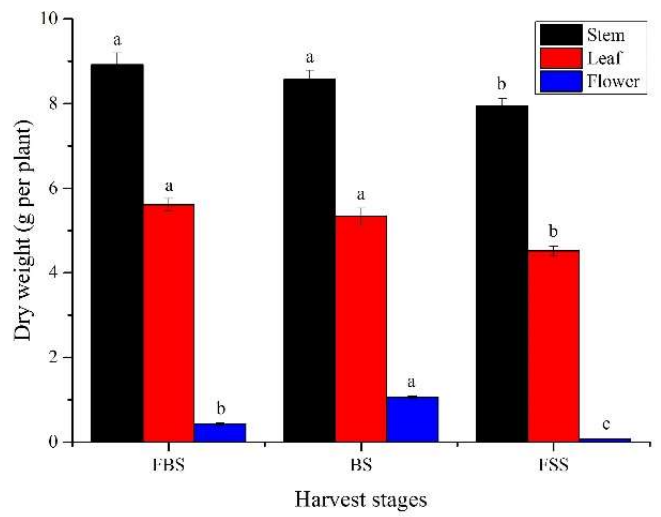

Figure 2. Aerial parts biomass of $H$. perforatum at different harvest stages. Values are on a per plant basis (mean $\pm \mathrm{SD}, \mathrm{n}=30)$. 


\subsection{Hypericin Content at Different Harvest Stages}

The representive chromatograms of Hyp quantification were shown in Figure 3. The Hyp content in aerial parts (stem, leaf and flower) was calculated based on peak area comparison with a reference standard. On a dry weight basis, the level of Hyp in flower reached the highest at BS, which is consistent with previous reports $[8,10]$, while the level of Hyp in stem and leaf exhibited a significant decrease from FBS to FSS (Figure 4.A). On a per plant basis, the level of Hyp in flower also exhibited the highest at BS, however, significant decreases in stem and leaf was observed from FBS to FSS. Due to large variation of the proportion of stem: leaf: flower (Figure 2), in a whole, the level of Hyp in aerial parts decreased from FBS to FSS, with reduction of 4.6- and 4.4-fold at FSS compared to FBS and BS, respectively (Figure 4.B).

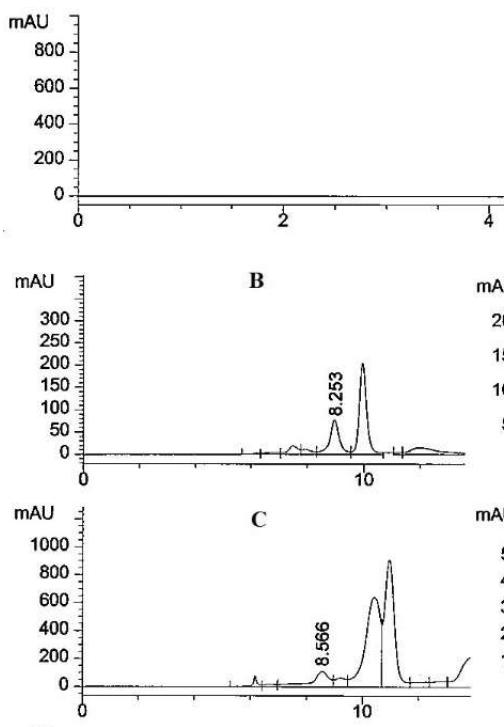

A
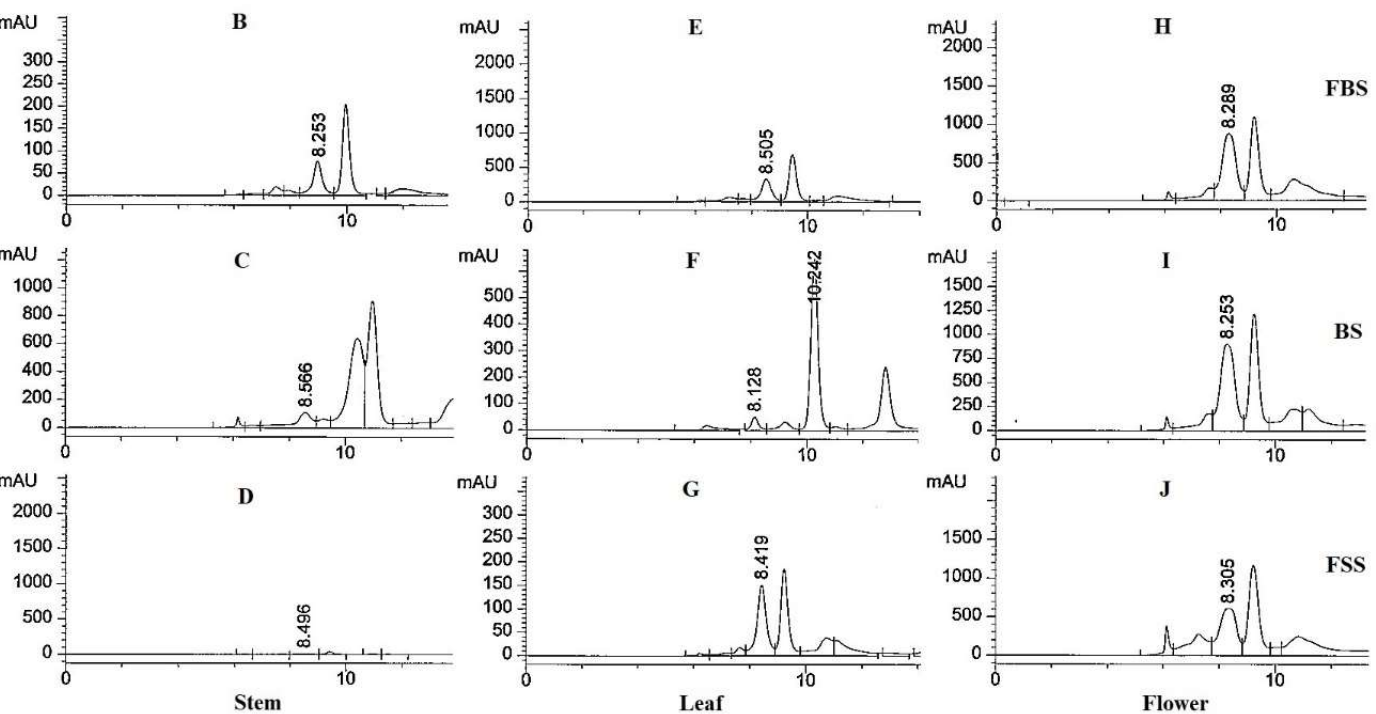

Figure 3. Representive chromatograms of Hyp quantification in aerial parts at different harvest stages. Image A represents standard Hyp at $1.0 \mathrm{mg} / \mathrm{mL}$ with injection $20 \mu \mathrm{L}$; images B, C, D, E, F, G, H, I and J represent stem, leaf and flower at FBS, BS and FSS.
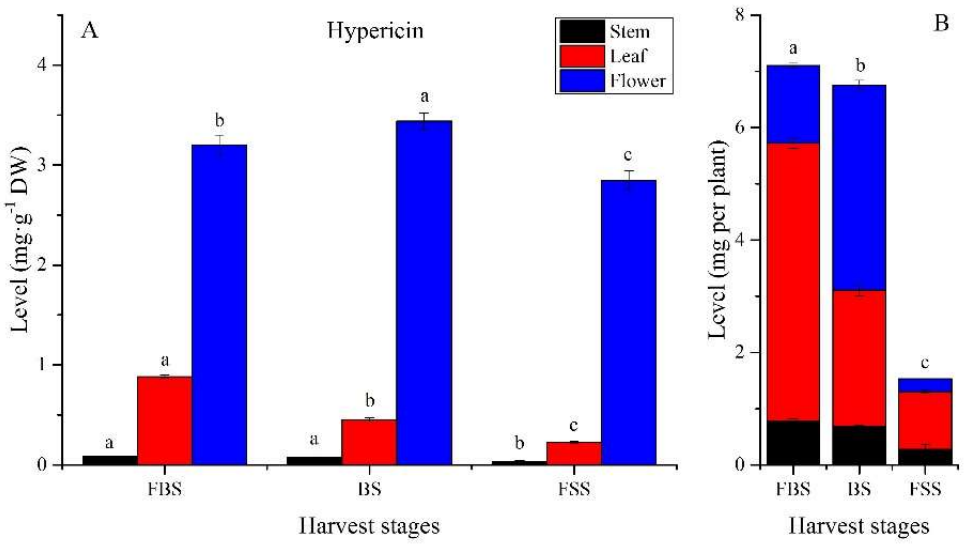

Figure 4. Hypericin (Hyp) content in aerial parts at different harvest stages. Images A and B represent Hyp content on dry weight and per plant basis, respectively (mean $\pm \mathrm{SD}, \mathrm{n}=30$ ). 


\subsection{Flavonoids and Polyphenols Contents at Different Harvest Stages}

The variation of flavonoids and polyphenols contents showed similar trends with Hyp (Figure 5 and Figure 6). Both flavonoids and polyphenols exhibited the highest levels in flower at BS, which is consistent with previous study that some flavonoids (pseudohypericin, isoquercetin, quercitrin and quercetin) and polyphenols (chlorogenic acid) peaked at BS in aerial parts [10], while decreases in stem and leaf from FBS to FSS were observed on a dry weight basis (Figure 5.A and Figure 6.A). Since the aerial parts biomass largely varied during harvest stages (Figure 2), as a result, both flavonoids and polyphenols contents decreased during harvest stages based on a per plant basis, with their reductions of 1.7- and 1.6-fold at FSS compared to FBS and BS, respectively (Figure 5.B and Figure 6.B).
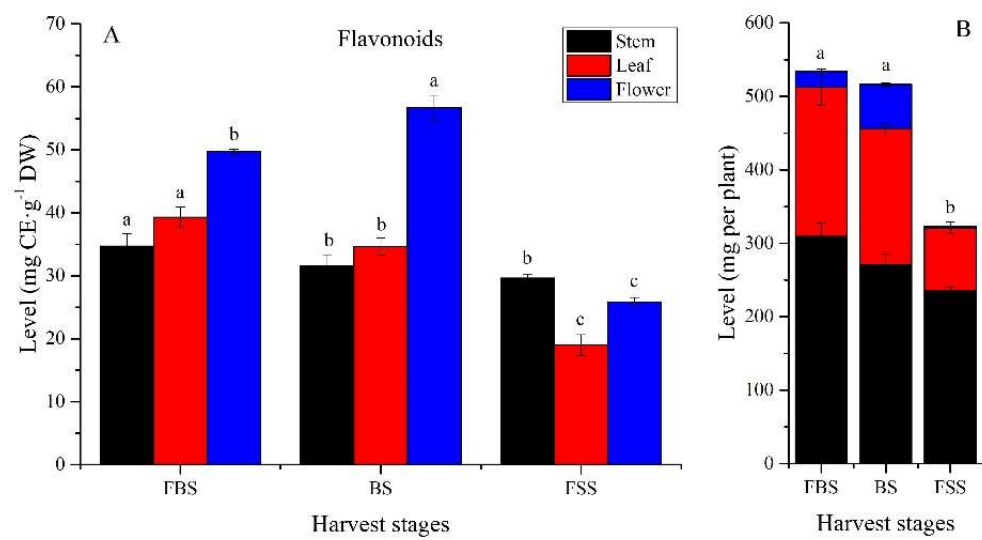

Figure 5. Flavonoids content in aerial parts at different harvest stages. Images A and B represent flavonoids content on dry weight and per plant basis, respectively (mean $\pm \mathrm{SD}, \mathrm{n}=30$ ).
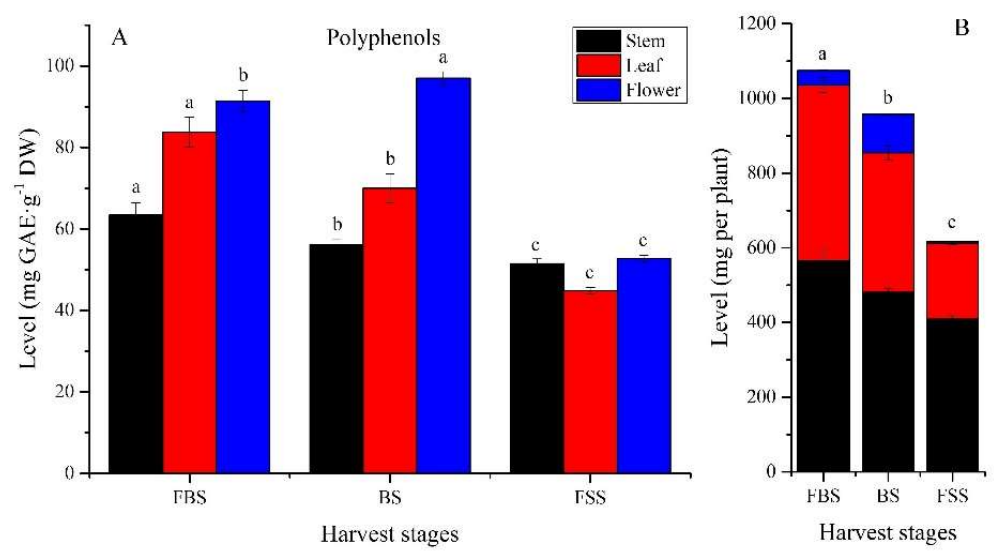

Figure 6. Polyphenols content in aerial parts at different harvest stages. Images A and B represent polyphenols content on dry weight and per plant basis, respectively (mean $\pm \mathrm{SD}, \mathrm{n}=30$ ).

Flavonoids and polyphenols compounds, as important categories of phytochemicals, have been considered to be major contributors to the antioxidant activity; and positive relationships of flavonoids and/or polyphenols contents with DPPH scavenging activity and/or FRAP value have been observed in many investigations $[16,24,25,27,28]$. In this study, the variation of flavonoids and polyphenols contents showed similar trends with DPPH scavenging activity and FRAP value, which suggested that the flavonoids and polyphenols in $H$. perforatum may play important roles in antioxidant capacity. Indeed, the constituents of flavonoids including: rutin, hyperoside, isoquercitrin, avicularin, quercitrin and quercetin as well as quercetin-3-O-rutinoside, galactoside and rhamnoside that exhibited free radical scavenging activity, metal-chelation activity and reactive oxygen quenching activity [29, 30]; and polyphenols, especially chlorogenic acid, showed high antioxidant activities including: DPPH and 
FRAP assays, NO scavenging, superoxide scavenging and lipid peroxidation, while phloroglucinols and naphthodianthrones showed no significant activity [30]. Above mentioned results indicated that the flavonoids and polyphenols in $H$. perforatum exhibited multi-antioxidant mechanisms, while the antioxidant mechanism of individual compound still need further study.

In conclusion, the levels of antioxidant capacity and bioactive compounds as well as aerial parts biomass were significantly affected by harvest stages, exhibiting a decreasing trend from floral budding stage (FBS) to fruit set stage (FSS). In brief, no significant difference in DPPH scavenging activity and FRAP value between FBS and blooming stage (BS) was observed; although the levels of hypericin (Hyp), flavonoids and polyphenols in flower reached the highest at BS on a dry weight basis, the levels of Hyp, flavonoids and polyphenols in aerial parts on a per plant basis exhibited a remarkable decrease from FBS to FSS, which indicated that the aerial parts should be harvested at FBS. These findings will provide useful information for bioactive compounds production and cultivation practice of $H$. perforatum.

\section{Acknowledgments}

This work was financially supported by Agriculture and Animal Husbandry of Gansu Province (GYC14-03, GNCX-2016-12), "Fuxi" Project of Gansu Agricultural University (Gaufx-02J04) and Collaborative and Innovative Science and Technology Team Supporting Program for University in Gansu Province, China (2016C-05).

\section{Competing Interests}

Authors have declared that no competing interests exist.

\section{Supporting Information}

Supporting Information accompanies this paper on http://www.acgpubs.org/RNP

\section{ORCID}

Ping Sun: 0000-0002-2017-8389

Tianlan Kang: 0000-0001-8528-787X

Hua Xing: 0000-0002-9699-1130

Zhen Zhang: 0000-0003-0519-1566

Delong Yang: 0000-0001-5370-1589

Jinlin Zhang: 0000-0002-3562-920X

Paul W. Paré: 0000-0003-1644-723X

Mengfei Li: $\underline{0000-0003-3035-4427}$

\section{References}

[1] G. D. Carlo, F. Borrelli, E. Ernest and A. A. Izzo (2001). St. John's Wort: Prozac from the plant kingdom, Trends Pharmacol. Sci. 22, 292-297.

[2] X. Kang, L. Ge, M. F. Li, Y. T. Gan, J. Y. Li and K. P. Wang (2016). Changes in physiological and biochemical characteristics, bioactive compounds and antioxidant capacity of Hypericum perforatum during introduction and acclimatization, Acta Prataculturae Sinica 25, 104-110.

[3] J. Barnes, L. A. Anderson and J. D. Phillipson (2001). St John's Wort (Hypericum perforatum L.): A review of its chemistry, pharmacology, and clinical properties, J. Pharm. Pharmacol. 53, 583-600.

[4] A. Nahrstedt and V. Butterweck (1997). Biologically active and other chemical constituents of the herb of Hypericum perforatum L, Pharmacopsychiatry 30, S129-S134. 
[5] K. Helmja, M. Vaher, T. Püssa, A. Orav, A. Viitak, T. Levandi and M. Kaljurand (2011). Variation in the composition of the essential oils, phenolic compounds and mineral elements of Hypericum perforatum L. growing in Estonia, Nat. Prod. Res. 25, 496-510.

[6] V. Jakovljević, M. Popović, N. Mimica-Dukić, A. Sabo and L. Gvozdenović (2000). Pharmacodynamic study of Hypericum perforatum L, Phytomedicine 7, 449-453.

[7] I. A. Southwell and C.A. Bourke (2001). Seasonal variation in hypericin content of Hypericum perforatum L. (St. John's Wort), Phytochemistry 56, 437-441.

[8] L. Ge, Y. Y. Yao, T. L. Kang, J. Y. Li, H. J. He, D. L. Yang and M. F. Li (2017). Changes in antioxidant capacity, main bioactive compounds in flower of Hypericum perforatum at different harvest stages and volatile constituents separation and identification, Acta Prataculturae Sinica 26, 66-74.

[9] D. P. Briskin, A. Leroy and M. Gawienowski (2000). Influence of nitrogen on the production of hypericins by St. John's Wort, Plant Physiol. Biochem. 38, 413-420.

[10] D. E. Gray, S. G. Pallardy, H. E. Garrett and G. E. Rottinghaus (2003). Effect of acute drought stress and time of harvest on phytochemistry and dry weight of St. John's Wort leaves and flowers, Planta Med. 69, 1024-1030.

[11] M. A. Couceiro, F. Afreen, S. M. A. Zobayed and T. Kozai (2006). Variation in concentrations of major bioactive compounds of St. John's Wort: effects of harvesting time, temperature and germplasm, Plant Sci. 170, 128-134.

[12] M. Azizi (2007). Change in content and chemical composition of Hypericum perforatum L. oil at three harvest time, J. Herbs Spices Med. Plants 13, 79-85.

[13] E. N. Frankel (1993). In search of better methods to evaluate natural antioxidants and oxidative stability in food lipids. Trends Food Sci. Technol. 4, 220-225.

[14] A. C. Mot, R. Silaghi-Dumitrescu and C. Sarbu C (2011). Rapid and effective evaluation of the antioxidant capacity of propolis extracts using DPPH bleaching kinetic profiles, FT-IR and UV-vis spectroscopic data, J. Food Compos. Anal. 24, 516-522.

[15] M. B. Arnao (2000). Some methodological problems in the determination of antioxidant activity using chromogen radicals: a practical case, Trends Food Sci. Technol. 11, 419-421.

[16] M. F. Li, P. W. Pare, J. L. Zhang, T. L. Kang, Z. Zhang, D. L. Yang, K. P. Wang and H. Xing (2018). Antioxidant capacity connection with phenolic and flavonoid content in Chinese medicinal herbs, Rec. Nat. Prod. 3, 239-250.

[17] L. K. Macdonaldwicks, L. G. Wood and M. L. Garg (2006). Methodology for the determination of biological antioxidant capacity in vitro: A review, J. Sci. Food Agr. 86, 2046-2056.

[18] M. C. Foti, D. A. Carmelo and C. Geraci (2004). Electron-transfer reaction of cinnamic acids and their methyl esters with the DPPH radical in alcoholic solutions, J. Org. Chem. 69, 2309-2314.

[19] W. Brand-Williams, M. E. Cuvelier and C. Berset (1995). Use of a free radical method to evaluate antioxidant activity, LWT-Food Sci. Tech. 28, 25-30.

[20] I. F. F. Benzie and J. J. Strain (1996). The ferric reducing ability of plasma (FRAP) as a measure of "antioxidant power": The FRAP assay, Anal. Biochem. 239, 70-76.

[21] M. F. Li, L. L. Zhou, D. L. Yang, T. T. Li and W. Li (2012). Biochemical composition and antioxidantcapacity of extracts from Podophyllumhexandrum rhizome, BMC Complem. Alterna. Med. 12, 263.

[22] J. D. Chi and M. Franklin (1999). Determination of hypericin in plasma by high-performance liquid chromatography, J. Chromatogr. B. 724, 195-198.

[23] C. M. Cantín, M. A. Moreno and Y. Gogorcena (2009). Evaluation of the antioxidant capacity, phenolic compounds, and vitamin C content of different peach and nectarine [Prunus persica (L.) Batsch] breeding progenies, J. Agr. Food. Chem. 57, 4586-4592.

[24] S. Li, S. K. Li, R. Y. Gan, F. L. Song, L. Kuang and H. B. Li (2013). Antioxidant capacities and total phenolic contents of infusions from 223 medicinal plants, Ind. Crop. Prod. 51, 289-298.

[25] R. Y. Gan, X. R. Xu, F. L. Song, K. Lei and H. B. Li (2010). Antioxidant activity and total phenolic content of medicinal plants associated with prevention and treatment of cardiovascular and cerebrovascular diseases, J. Med. Plants Res. 4, 2438-2444.

[26] H. Jaberian, K. Piri and J. Nazari (2013). Phytochemical composition and in vitro antimicrobial and antioxidant activities of some medicinal plants, Food Chem. 136, 237-244.

[27] Y. Z. Cai, Q. Luo, M. Sun and H. Corke (2004). Antioxidant activity and phenolic compounds of 112 Chinese medicinal plants associated with anticancer, Life Sci. 74, 2157-2184.

[28] X. Wang, M. Wang, J. Cao, Y. Wu, J. Xiao and Q. Wang (2017). Analysis of flavonoids and antioxidants in extracts of ferns from Tianmu mountain in Zhejiang province (China), Ind. Crop. Prod. 97, 137-145. 
[29] Y. P. Zou, Y. H. Lu and D. Z. Wei (2004). Antioxidant activity of a flavonoid-rich extract of Hypericum perforatum L. in vitro, J. Agric. Food Chem. 52, 5032-5039.

[30] D. Z. Orčić, N. M. Mimica-Dukić, M. M. Francišković, S. S. Petrović and E. D. Jovin (2011). Antioxidant activity relationship of phenolic compounds in Hypericum perforatum L., Chem. Cent. J. 5, 34.

\section{(C) 2018 ACG Publications}

